\title{
Rapidly quantitative antimicrobial susceptibility testing for Klebsiella pneumoniae using Matrix- Assisted Laser Desorption Ionization-Time of Flight Mass Spectrometry under optimal conditions
}

\author{
Anran Zhang \\ Tianjin Medical University General Hospital \\ Qianqian Chen \\ Tianjin Medical University General Hospital \\ Yulan Dong \\ Tianjin Medical University General Hospital \\ Bin Tian \\ Tianjin Medical University General Hospital \\ Jing Li \\ Tianjin Medical University General Hospital \\ Zhidong Hu ( $\nabla$ hzd27@qq.com ) \\ Tianjin Medical University General Hospital
}

\section{Research Article}

Keywords: Klebsiella pneumoniae, MALDI-TOF MS, RAST, optimization conditions

Posted Date: November 18th, 2021

DOI: https://doi.org/10.21203/rs.3.rs-1048817/v1

License: (a) (1) This work is licensed under a Creative Commons Attribution 4.0 International License.

Read Full License 


\section{Abstract \\ Background}

Klebsiella pneumoniae infections, especially Carbapenem-resistant Klebsiella pneumoniae (CRKP), have become an "Urgent Threats" with high morbidity and mortality. Therefore, rapidly determining of the susceptibility and timely choosing an appropriate antibiotic were the important premises of the treatment of Klebsiella pneumoniae infections. The present study was first to explore the fitness of matrix-assisted laser desorption ionization-time of flight mass spectrometry (MALDI-TOF MS) in quantitative rapid antimicrobial susceptibility testing (RAST) with optimal conditions.

\section{Methods}

Firstly, we optimized the methodology based on the formic acid extraction method. Taking broth microdilution method (BMD) as a reference method, we utilized 25 -fold, 50-fold and 100 -fold dilutions matching with appropriate incubation time to evaluate the consistency of minimum inhibition concentration (MIC) values derived from BMD and MALDI-TOF MS. Finally, the performances of optimal dilution and incubation time were verified through the reproducibility on different three days.

\section{Results}

Directly incubated in the EP tubes and skipped the washing step can not only simplify the procedure, but reduce the unnecessary loss of bacteria biomass to improve sensitivity. The optimal volumes of $70 \%$ formic acid/100\% acetonitrile (FA/ACN) and supernatant on the target plate were $3 \mu \mathrm{L}$ and $2 \mu \mathrm{L}$, respectively. Comparing the different combination, 25 -fold dilution of the $0.5 \mathrm{McFarland}$ suspension and $2 \mathrm{hr}$ incubation time resulted in best performance. The consistency between the MS-MIC and corresponding BMD-MIC values in CRKP and Carbapenem-susceptible Klebsiella pneumoniae (CSKP) strains accounted for $76.67 \%$ and $66.67 \%$, respectively, which generated the substantial agreements between MS-MIC and BMD-MIC values for CRKP strains (Kappa=0.643) and the moderate agreements for CSKP strains (Kappa=0.476). Finally, the MALDI-TOF MS-based RAST showed a well repeatability.

\section{Conclusion}

Considerable potentials were demonstrated for universal and mechanism-indepent RAST by MALDI-TOF MS with optimal conditions, which strengthened its application in accelerating reporting time and clinical diagnosis.

\section{Bcakground}


Klebsiella pneumoniae was an opportunistic pathogen of clinical importance and the CRKP was still listed as an "Urgent Threats" in the 2019 Antibiotic Resistance Threats Report by the Centers for Disease Control and Prevention (CDC)[1], which was one of the most prioritized threats of antibiotics resistance in the healthcare facilities, warranting urgent and aggressive action. The promptly increasing resistance and the resulting high mortality of CRKP that admit of no optimism in many countries[2-4]presented a global challenge. In particular, the mortality of the bloodstream infections (BSIs) caused by CRKP was as high as $71.9 \%[5]$, three times higher than infections in other sites. Therefore, rapidly determining of the MIC values and timely choosing an appropriate antibiotic were the important premises of the treatment of patients with Klebsiella pneumoniae infections.

However, the reporting time of the routine AST, such as E-test, disk diffusion, BMD and VITEK-2 system, can take up to at least one day[6], because these routine phenotypic assays required extensive proliferation to reach detection level. Technically, MALDI-TOF MS[7], integrated microfluidic device[8], biological small-molecule assays[9], diffraction-based method[10] and other innovations for RAST were flourishing to shorten the turnaround time and accelerate clinical diagnostics. The above MALDI-TOF MS not only was the first-line tool[11] to identify pathogens with simplicity, speediness, cost-efficiency, highthroughput and high-resolution, but has been developed for rapid detection of antimicrobial resistance. Furtherly, MALDI-TOF MS-based RAST about Klebsiella pneumoniae were mainly divided into the following three approaches:

1. Identify the MS peaks of specific protein associated with resistance. The strong correlation between bla ${ }_{K P C}$-harboring pKpQIL-like plasmids[12-13]and the $11,109 \mathrm{~m} / \mathrm{z}$ MS peak has been confirmed with excellent specificity (100\%) and sensitivity (85.1\%)[14], which succeeded in the real-time detection of KPC-producing CRKP during the routine clinical identification. Apart from carbapenemases, loss or modification of the major outer membrane porins (OMPs) OmpK35 and OmpK36 also aggravated the carbapenem-resistance of Klebsiella pneumoniae. Hu et al.[15] found three characteristic peak (ca. $36,000 \mathrm{~m} / \mathrm{z}, 38,600 \mathrm{~m} / \mathrm{z}$ and $37,000 \mathrm{~m} / \mathrm{z}$ ) corresponding to the OmpA, OmpK36 and OmpK35 in CSKP by MALDI-TOF MS within half an hour, which proved MALDI-TOF MS had a better ability of identifying porin-deficient strains than sodium dodecylsulfate-polyacrylamide gel electrophoresis (SDS-PFGE). In addition, five highly expressed characteristic peptide peaks of serum biomarkers, including $1349.8 \mathrm{~m} / \mathrm{z}, 2091.3 \mathrm{~m} / \mathrm{z}, 2908.2 \mathrm{~m} / \mathrm{z}, 4102.1 \mathrm{~m} / \mathrm{z}$, and $8129.5 \mathrm{~m} / \mathrm{z}[16]$, were chosen to distinguish CRKP-BSI from normal healthy controls.

2. Analyse the MS peak profiles of the antibiotic hydrolysis and monitor the corresponding products. The first application of MALDI-TOF MS-based antibiotic hydrolysis assay was visualized the hydrolysis of the $\beta$-lactam ring[17] by the reduction of the peak corresponding to the antibiotic and appearance of peaks of the hydrolysis products. In a similar way, after 30 min-3hrincubation with carbapenemase-producing Klebsiella pneumoniae, MS peaks of ertapenem and its salt adducts peaks (476.5 Da, 498.5 Da, 520.5 Da) would disappeared, to be replaced by the hydrolyzed products(472.5 $\mathrm{Da}, 538.5 \mathrm{Da})[18]$. Recently, some studies utilized the hydrolysis index $(\mathrm{HI})[19], \mathrm{MS}$ 
ratio[20] and logarithm of the quantification of resistance (LogRQ) values[21] and other algorithms to semi-quantitatively differentiate sensitive and resistant strains.

3. Compare the bacterial growth by MALDI-TOF MS in the presence or absence of antibiotic. The most advantage of growth-based RAST was the universality, which independently from the specific resistance mechanism. For Klebsiella pneumoniae, Lange et al.[22] firstly developed the MALDI Biotyper antibiotic susceptibility test rapid assay (MBT-ASTRA), extracting bacterial protein after incubation with meropenem breakpoint concentration, adding an internal standard, normalizing the maximum peak and calculating the relative growth value to semi-quantitatively detect the resistance. And the newly developed direct-on-targt microdroplet growth asssy (DOT-MGA)[23] was also growthbased RAST but with $6 \mu \mathrm{L}$ micro-droplet and more simplified processing process.

Recently studies have achieved qualitative[24-25] and semi-quantitative AST[26] by MALDI-TOF MS, but there were few studies conducted quantitative tests. Here, we aimed at developing quantitative RAST that are based on MALDI-TOF MS technology implemented in the VITEK MS instrument with optimized assay conditions. According to European Committee on Antimicrobial Susceptibility Testing (EUCAST) or Clinical Laboratory Standards Institute (CLSI) recommendations[27], we adopted the growth-based phenotypic RAST to determine the susceptibility of the Klebsiella pneumoniae, independently from some specific resistance mechanisms. And to our knowledge, this was the first study to evaluate the in vitro diagnostic (IVD) mode of the VITEK MS under the routine conditions and the existing parameter settings.

\section{Results}

\section{Optimal conditions for MALDI-TOF MS-based quantitative RAST.}

Compared with previously published study[25], (i) we directly incubated the diluted bacterial suspension into the $200 \mu \mathrm{L}$ EP tubes with or without imipenem (IPM), rather than incubating in the flat-bottomed 96well plates and transferring into the Eppendorf (EP) tubes and (ii) we skipped the washing step, although some studies washed the precipitable bacteria with pure water and $70 \%$ alcohol after the first time centrifugation. We found that no washing had no significant effect on strain identification (Fig. 1). Conversely, transferring into the EP tubes and washing the precipitable bacteria would cause unnecessary loss of bacteria, which led to MIC values identified by MALDI-TOF MS (MS-MIC) decline to lower concentrations. Further research demonstrated that adding $3 \mu \mathrm{L}$ of FA/ACN was enough to split bacteria and extract protein in the preprocessing step. Excessive addition may diluted the protein concentration to reduce sensitivity. And the optimal volume of the supernatant on the VITEK MS target plate was $2 \mu \mathrm{L}$. Using this optimized combination, the validity for the 25 -fold, 50 -fold and 100 -fold dilution reached $100 \%$ in the $2 h r, 3 h r$ and $3 h r$, respectively.

\section{Consistency between MS-MIC and BMD-MIC.}

The results of the BMD showed that the ranges of the MIC values in CRKP and CSKP strains were 32ख256 $\mu \mathrm{g} / \mathrm{mL}\left(\mathrm{MIC}_{50 / 90}, 128 / 256 \mu \mathrm{g} / \mathrm{mL}\right.$ ) and $0.25 \llbracket 2 \mu \mathrm{g} / \mathrm{mL}\left(\mathrm{MIC}_{50 / 90}, 1 / 1 \mu \mathrm{g} / \mathrm{mL}\right)$, respectively. In this study, 
all strains were firstly tested in the 100 -fold dilution (final concentration $\varangle 5 \times 10^{5} \mathrm{CFU} / \mathrm{mL}$ ) as the same as BMD with a short incubation time of $4 \mathrm{hr}$. But there were only fair or moderate agreement between the MS-MIC and BMD-MIC, with the kappa values of 0.316 (Tab. 1) in CRKP strains and 0.406 (Tab. 2) in CSKP strains. In CRKP strains, 50\% (15/30) of the MS-MIC values were at least one concentration gradients lower than the corresponding BMD-MIC, including $13.33 \%(4 / 30)$ of the MS-MIC values even reduced two concentration gradients. In CSKP strains, shifts of MS-MIC values to lower concentration gradients was relieved and only 30\% (9/30) of MS-MIC values expressed lower concentrations. However, the MS-MIC values $(2,1,0.5 \mu \mathrm{g} / \mathrm{mL}$, respectively) of three CSKP strains were one concentration gradient higher than its BMD-MIC values $(1,0.5,0.25 \mu \mathrm{g} / \mathrm{mL})$.

Subsequently, we applied the initial inoculum size, which was higher than CLSI-recommended inoculum $\left(5 \times 10^{5} \mathrm{CFU} / \mathrm{mL}\right.$ with an acceptable range of $2 \times 10^{5} \mathrm{CFU} / \mathrm{mL}$ to $\left.8 \times 10^{5} \mathrm{CFU} / \mathrm{mL}\right)$ as a precautionary measure to reduce the shifting to a lower concentration gradient and compensate the reduction in incubation time. In the pre-experimental phase, we confirmed the dilution of 50 -fold ( final concentration $\nabla 1 \times 10^{6} \mathrm{CFU} / \mathrm{mL}$ ) and 25 -fold ( final concentration $\otimes 2 \times 10^{6} \mathrm{CFU} / \mathrm{mL}$ ), which initial biomass could not reach the limit of detection (LOD) of MALDI-TOF MS, but could after a short-term incubation. After verification, 25 -fold dilution of the $0.5 \mathrm{McF}$ arland suspension and $2 \mathrm{hr}$ incubation time resulted in best performance under the optimized formic acid extraction method. There were substantial agreements between the MIC values derived from MALDI-TOF MS and BMD for CRKP strains (Kappa=0.643) and moderate agreements for CSKP strains (Kappa=0.476). For CRKP strains, 76.67\% (23/30) of the MS-MIC values were equal to corresponding BMD-MIC values. Of the remaining seven strains, the MS-MIC values of five strains were one $(n=4)$ or two $(n=1)$ concentration gradients lower than its BMD-MIC values and two strains were higher one concentration gradients. BMD-MIC values of the former mainly focused on the high concentration gradient $(256,128,128,128,128 \mu \mathrm{g} / \mathrm{mL}$, respectively), while the latter was lower $(64,32 \mu \mathrm{g} / \mathrm{mL}$, respectively). Along with the time extended to $3 \mathrm{hr}$, CRKP strains had higher MS-MIC values compared with BMD-MIC values, which accounted for 33.33\%(10/30). For CSKP strains, although the consistency of incubation at 25 -fold dilution for $2 \mathrm{hr}$ cannot hold a candle to the consistency of CRKP strains, it created the highest consistency of the $66.67 \%(20 / 30)$ in all protocols. And the main reason of inconsistency was that the MS-MIC values transferred a higher concentration gradient but no major error (ME, false resistance) in susceptibility interpretation occurred. In addition, the consistency of the MIC values detected by MALDI-TOF MS and BMD of the incubation at 50-fold dilution for $4 \mathrm{hr}$ in CRKP strains was nearly the same as that at 25 -fold for $2 h r$ but with a large time cost. However, shortened the time to $3 h r$, the consistency in CRKP strains suddenly dropped to $46.67 \%$ and the consistency showed no statistical significance $(P=0.584)$ in CSKP strains. 
Table 1

The consistency of the MIC values detected by VITEK MS and BMD in CRKP strains

\begin{tabular}{|llllll|}
\hline Dilution & $\mathbf{1 : 1 0 0}$ & $\mathbf{1 : 5 0}$ & & $1: 25$ & \\
\hline Incubation time & $4 \mathrm{~h}$ & $3 \mathrm{~h}$ & $4 \mathrm{~h}$ & $2 \mathrm{~h}$ & $3 \mathrm{~h}$ \\
\hline MS-MIC=BMD- & $50.00 \%$ & $46.67 \%(14 /$ & $73.33 \%(22 /$ & $76.67 \%$ & $66.67 \%(20 /$ \\
MIC & $(15 / 30)$ & $30)$ & $30)$ & $(23 / 30)$ & $30)$ \\
\hline MS-MIC<BMD-MIC & $50.00 \%$ & $30.00 \%(9 /$ & $10.00 \%(3 /$ & $16.67 \%(5 /$ & $0.00 \%(0 / 30)$ \\
& $(15 / 30)$ & $30)$ & $30)$ & $30)$ & \\
\hline MS-MIC>BMD-MIC & $0.00 \%(0 / 30)$ & $23.33 \%(7 /$ & $16.67 \%(5 /$ & $6.67 \%(2 /$ & $33.33 \%(10 /$ \\
& & $30)$ & $30)$ & $30)$ & $30)$ \\
\hline $\begin{array}{l}\text { Cohen's kappa } \\
\text { values }\end{array}$ & $0.316 \pm 0.117$ & $0.241 \pm 0.121$ & $0.582 \pm 0.127$ & $0.643 \pm 0.117$ & $0.508 \pm 0.111$ \\
$P$ & & & & & $<0.001$ \\
\hline
\end{tabular}

Table 2

The consistency of the MIC values detected by VITEK MS and BMD in CSKP strains

\begin{tabular}{|llll|}
\hline Dilution & $1: 100$ & $1: 50$ & $1: 25$ \\
\hline Incubation time & $4 \mathrm{~h}$ & $3 \mathrm{~h}$ & $2 \mathrm{~h}$ \\
\hline MS-MIC=BMD-MIC & $60.00 \%(18 / 30)$ & $36.67 \%(11 / 30)$ & $66.67 \%(20 / 30)$ \\
\hline MS-MIC<BMD-MIC & $30.00 \%(9 / 30)$ & $10.00 \%(3 / 30)$ & $0.00 \%(0 / 30)$ \\
\hline MS-MIC>BMD-MIC & $10.00 \%(3 / 30)$ & $53.33 \%(16 / 30)$ & $33.33 \%(10 / 30)$ \\
\hline Cohen's kappa values & $0.406 \pm 0.127$ & $0.058 \pm 0.115$ & $0.476 \pm 0.134$ \\
$P$ & $<0.001$ & 0.584 & $<0.001$ \\
\hline
\end{tabular}

\section{Reproducibility Of The Maldi-tof Ms-based Quantitative Rast}

To evaluate reproducibility of the MALDI-TOF MS-based quantitative RAST, we tested the MS-MIC values of the incubation at 25-fold dilution for 2 hrof all strains on different three days (Fig. 2). The consistency between the MS-MIC and BMD-MIC in CRKP strains accounted for $76.67 \%, 66.67 \%$ and $76.67 \%$, respectively, and CSKP strains $66.67 \%, 63.33 \%$ and $70.00 \%$, respectively. Inconsistent results of CRKP strains in three repeatable tests mainly shifted to lower concentrations and CSKP higher concentrations. Meanwhile, the results of three repeatability tests indicated that the MS-MIC value distributions of CSKP strain was more stable than that of CRKP strain (Fig. 3).

\section{Discussion}


MBT-ASTRA[22] and DOT-MGA[23] were the emerging technologies of the MALDI-TOF MS-based qualitative AST, but the former was limited to the specific brand of equipment to calculate the MS peak area under curve (AUCs) with a classification between susceptibility and resistance. The application of the latter was universality and simplicity, but merely 'touching' of the microdroplets with a tissue wipe to removed the broth would exert an influence on the results. In this study, we combined the MBT-ASTRA and DOT-MGA and successfully applied the MALDI-TOF MS to quantitatively detect MIC values of 30 CRKP strains and 30 CSKP strains under the optimized conditions within $2 \mathrm{hr}$ and further verify the reproducibility.

Compared with previous studies, we optimized the sample processing process. One of the reasons why BMD used flat-bottomed 96-well plates was facilitated to visual reading the turbidity after the 18-20 hr incubation to identity the MIC values. However, the incubation time of the MALDI-TOF MS-based AST was too short to generate visual differences between the bacterial suspensions incubated with different concentration of IPM. Directly incubated in the EP tubes and skipped the washing step can not only simplify the procedure and and shorten the reporting time, but reduce the unnecessary loss of bacteria biomass during the processing stage, which prevented the MS-MIC values from declining to a lower concentration and improved the sensitivity.

The prospect of the growth-based phenotypic RAST was partly hindered by the duration of the lag phase[28], which was the time needed for strains to cope with the constraints of their environment before proliferation. Within a population, the intensity of respiration repression[29] caused the considerable heterogeneity in the lag duration and growth rate between individuals. On the other hand, it was found that the main problem of the incubation with 100-fold dilution of BMD for a short time was the phenomenon of delayed phenotypic resistance. Some strains can express susceptibility at the early stages of incubation, caused the shifts of MS-MIC values to lower concentration gradients. After a long incubation, the strains gradually grew to express the correct MIC values in the presence of high concentrations of antibiotics. In this study, $50 \%(n=15)$ of the CRKP strains and $30 \%(n=9)$ of the CSKP strains were significantly affected by this phenomenon. Wang et al.[30] conducted a RAST for Klebsiella pneumoniae using a suspension of $5 \times 10^{5} \mathrm{CFU} / \mathrm{mL}$ bacterial cells incubating for 4 hr by MALDI TOF MS Research-User-Only (RUO) mode. The $43.33 \%$ of the CRKP strains and $13.33 \%$ of the CSKP strains were influenced by the phenomenon of delayed phenotypic resistance to show lower MIC values detected by MALDI-TOF MS compared to those derived from the BMD. The differences of inconsistencies possibly attributed to the acquisition system, algorithm and identification rules[31] of RUO and IVD mode respectively. At the same time, $10 \%(n=3)$ of the CSKP strains showed an increase in initial biomass. Our finding was consistent with $\mathrm{Li}$ et al.[25], who indicated that $8.60 \%$ of the susceptibility strains presented the MS-MIC values higher than the corresponding MIC values.

Inoculum effect (IE) [32], i.e. the initial inoculum of bacteria applied on AST may affected the interpretation of MIC values, demonstrated that minor, allowable deviations in inoculum would change the MIC values and thereby potentially affect therapeutic decision points. Therefore, determining the initial inoculum and matched incubation time of bacteria were key factors of implementing MALDI-TOF 
MS-based quantitative RAST. Compared with incubation with 100 -fold dilution for 4 hr, incubation with 25fold dilution for $2 \mathrm{hr}$ greatly improved the turnaround times and effectively reduced the inconsistency caused by the phenomenon of delayed phenotypic resistance in CRKP strains and CSKP strains by $33.33 \%(n=10)$ and $30.00 \%(n=9)$, respectively. However, improving the initial inoculum size inevitably led to an increase of $23.33 \%(n=7)$ in the inconsistency of CSKP strains. Finally, the limitation in our study was the MIC values of CRKP strains not fell on the breakpoints for resistant categories, which can not obtain a complete resistance profile of IPM.

\section{Conclusion}

With the development of technology and clinical databases, the fitness of MALDI-TOF MS for clinical microbiological applications will further expand, especially in RAST. There were considerable potentials for MALDI-TOF MS to provide universal, rapid and mechanism-indepent AST with the optimal conditions, which appeared feasible to drastically accelerate the clinical diagnostics and treatment .

\section{Methods}

\section{Bacterial strains}

A total of 60 random non-replicate clinical Klebsiella pneumoniae strains were collected from the blood of BSI patients at a tertiary hospital in Tianjin, China, between Jan 2018 and Jun 2021, including 30 CRKP and 30 CSKP. Klebsiella pneumoniae clinical strains were identified using VITEK MS (Biomérieux, France) and the AST was performed using an automated VITEK 2 Compact Antimicrobial Susceptibility card (Biomérieux, France). Based on the PCR results, all CRKP strains were indentified as carbapenemase producer and were positive for bla $a_{K P C}(\mathrm{n}=29)$ and $b / a_{N D M}(\mathrm{n}=1)$, corresponded to the phenotypic expression of resistance to imipenem (IPM).

\section{Reference resistance determination}

According to CLSI recommendations[27], MIC values were determined by BMD. Briefly, $0.5 \mathrm{McF}$ standard suspension was diluted 100 -fold (to give a final CFU/mL of $\otimes 1 \times 10^{6}$ ) by cation-adjusted Mueller-Hinton broth (CAMHB) and were inoculated triplicately into equal $100 \mu \mathrm{L}$ of CAMHB in flat-bottomed 96well plates (Corning, USA) containing two-fold serial dilutions of IPM giving final concentrations ranging from 256 to $0.125 \mu \mathrm{g} / \mathrm{mL}$. Wells with no IPM and wells with CAMHB alone were served as the positive and negative controls, respectively. Before and after incubation at $37^{\circ} \mathrm{C}$ overnight, the optical density (OD) value of each well was measured by a micro-plate reader (Biotek Synergy HT, USA) at $600 \mathrm{~nm}$. The BMD-MIC values was considered as the lowest concentration of the IPM that completely inhibited the bacterial growth with the OD value increase $\leq 0.050[33]$.

\section{Selection of the optimal conditions}


Comparative study of the optimal conditions for MALDI-TOF MS-based quantitative RAST included the volume of FA/ACN, the volume of supernatant spotted on the target plate, incubation time and dilution. Firstly, we optimized the operation and methodology based on the formic acid extraction method, which was recommended prior to MALDI-TOF MS analysis. The volume of FA/ACN and supernatant spotted on the VITEK MS target plate were determined to optimize test performance from $3 \mu \mathrm{L}, 5 \mu \mathrm{L}, 10 \mu \mathrm{L}$ and $1 \mu \mathrm{L}$, $2 \mu \mathrm{L}$, respectively. Subsequently, referring to the preliminary experimental results, a $0.5 \mathrm{McF}$ standard suspension was respectively diluted 25 -fold, 50 -fold and 100 -fold and inoculated into equal $100 \mu \mathrm{L}$ CAMHB without IPM. After incubated for $1,2,3,4 \mathrm{hr}$ at $37^{\circ} \mathrm{C}$, the appropriate incubation time of each dilution was preliminary identified when the validity reach $100 \%$, which was defined as the percentage of strains were successfully identified by the MALDI-TOF MS.

\section{MALDI-TOF MS-based quantitative RAST}

1. VITEK MS-based analysis. Each dilution was detected in the same way as the BMD with an established incubation time. Different from BMD, bacterial suspensions were directly incubated in $200 \mu \mathrm{L}$ Eppendorf (EP) tube and centrifuged at $13,000 \mathrm{~g}$ for $2 \mathrm{~min}$ [34]. Subsequently, discarded the supernatant, lysised the precipitable bacteria by the optimal volume of $70 \%$ formic acid for $10 \mathrm{~min}$ and extracted protein by the equal volume of $100 \%$ acetonitrile. Centrifuged, spotted optimal volume of the supernatant on the VITEK MS target plate, dried and overlaid with $1 \mu \mathrm{L}$ of a-cyano-4hydroxy-cinnamic acid (a-CHCA). After desiccation, the extractions were analyzed by the IVD mode on the VITEK MS (bioMérieux, France) calibrated with a quality-control strain of Escherichia coli ATCC8739 at a laser frequency of $200 \mathrm{~Hz}$, in the mass range of 2,000-20,000 Da.

2. Data capture and analysis. Data analysis was performed using the software Myla 3.2.0 (Biomérieux, France). The growth of bacteria treated with a certain concentration of IPM was quantified by the confidence level intended for identification. If the strains was not significantly inhibited at a certain concentration, the strains will be successfully identified with the confidence level of $\geq 90 \%$. Conversely, confidence level of $<90 \%$ or non-identification was interpreted as growth inhibition. The MS-MIC values were considered as the lowest concentration of the IPM at which confidence level was $<90 \%$ or had no identification.

\section{Statistical Analysis}

The percentage of observed agreements and the Cohen's kappa coefficient with its corresponding 95\% interval confidence were used to evaluate the consistency between the MALDI-TOF MS-based quantitative RAST and BMD of data and the reproducibility of the optimal combination between dilution and incubation time. Kappa values were stratified as follow: $0.8<$ Kappa $\leq 1$ indicated almost perfect agreement, $0.60<$ Kappa $\leq 0.80$ substantial agreement, $0.4<$ Kappa $\leq 0.60$ moderate agreement, $0.20<$ Kappa $\leq 0.4$ fair agreement and $0<K a p p a \leq 0.20$ slight agreement[35]. Statistical significance was defined as $<0.05$ in a two-tailed test. All statistical analyses were performed using the SPSS 23.0.

\section{Abbreviations}


matrix-assisted laser desorption ionization-time of flight mass spectrometry (MALDI-TOF MS)

rapid antimicrobial susceptibility testing (RAST)

broth micro-dilution method (BMD)

minimum inhibition concentration (MIC)

$70 \%$ formic acid $/ 100 \%$ acetonitrile (FA/ACN)

Carbapenem-resistant Klebsiella pneumoniae (CRKP)

Carbapenem-susceptible Klebsiella pneumoniae (CSKP)

Centers for Disease Control and Prevention (CDC)

bloodstream infections (BSIs)

outer membrane porins (OMPs)

sodium dodecylsulfate-polyacrylamide gel electrophoresis (SDS-PFGE)

hydrolysis index (HI)

logarithm of the quantification of resistance (LogRQ)

MALDI Biotyper antibiotic susceptibility test rapid assay (MBT-ASTRA)

direct-on-targt microdroplet growth asssy (DOT-MGA)

European Committee on Antimicrobial Susceptibility Testing (EUCAST)

Clinical Laboratory Standards Institute (CLSI)

in vitro diagnostic (IVD)

imipenem(IPM)

Eppendorf (EP)

MIC values identified by MALDI-TOF MS (MS-MIC)

limit of detection (LOD)

major error (ME)

area under curve (AUCs) 
Research-User-Only (RUO)

Inoculum effect (IE)

cation-adjusted Mueller-Hinton broth (CAMHB)

the optical density (OD)

a-cyano-4-hydroxy-cinnamic acid (a-CHCA)

\section{Declarations}

\section{Authors' contributions}

Anran Zhang conceived the experimen design, collected cilinical strains, carried out the laboratory tests and sorted out the results; Anran Zhang and Qianqian Chen wrote the first drafts of the manuscript; Yulan Dong, Bin Tian, Jing Li and Zhidong Hu revised the manuscript. All authors read and approved the final manuscript.

\section{Funding}

This research did not receive any specific grant from funding agencies in the public, commercial, or notfor-profit sectors.

\section{Availiability of data}

Data sets for this study are available on reasonable request from corresponding authors.

\section{Ethical Approval}

The experiment has been approved by the Ethics Committee of Tianjin Medical University General Hospital (Ethical NO. IRB2021-WZ-151). All methods were performed in accordance with the relevant guidelines and regulations by including a statement in the Ethics approval and consent to participate section.

\section{Consent to participate}

The patient's written informed consent was obtained in accordance with the Declaration of Helsinki.

\section{Consent for publication}

Published written informed consent can be obtained from all participants.

\section{Conflict of interest}

The authors declared that they had no conflict of interest. 


\section{References}

1. K Sameer S. Key Takeaways From the U.S. CDC's 2019 Antibiotic Resistance Threats Report for Frontline Providers. Critical care medicine. 2020; 48(7):939-945.

https://doi.org/10.1097/CCM.0000000000004371

2. Brolund A, Lagerqvist N, Byfors $S$, et al. Worsening epidemiological situation of carbapenemaseproducing Enterobacteriaceae in Europe, assessment by national experts from 37 countries, July 2018. Eurosurveillance. 2019; 24(9). https://doi.org/10.2807/1560-7917.ES.2019.24.9.1900123

3. Kazmierczak KM, Karlowsky JA, de Jonge BLM, et al. Epidemiology of Carbapenem Resistance Determinants Identified in Meropenem-nonsusceptible Enterobacterales collected as part of a Global Surveillance Program, 2012-2017. Antimicrobial agents and chemotherapy. 2021;65(7).https://doi.org/10.1128/AAC.02000-20

4. Zhiyong Z, Anhua W, Bijie H. Infection Control in the Era of Antimicrobial Resistance in China: Progress, Challenges, and Opportunities. Clinical infectious diseases: an official publication of the Infectious Diseases Society of America. 2020; 71(4): S372-S378. https://doi.org/10.1093/cid/ciaa1514

5. Maraolo AE, Silvia C, Adriano G, et al. The Impact of Carbapenem Resistance on Mortality in Patients With Klebsiella Pneumoniae Bloodstream Infection: An Individual Patient Data Meta-Analysis of 1952 Patients. Infectious diseases and therapy. 2021; 10(1): 541-558.

https://doi.org/10.1007/s40121-021-00408-8

6. Jae HH,Joon SD, Jin SH, et al. Performance evaluation of the QMAC-dRAST for staphylococci and enterococci isolated from blood culture: a comparative study of performance with the VITEK-2 system. The Journal of antimicrobial chemotherapy. 2018;73(5), 1267-1271.

https://doi.org/10.1093/jac/dky015

7. Idelevich E A, Becker K. How to accelerate antimicrobial susceptibility testing. Clinical microbiology and infection: the official publication of the European Society of Clinical Microbiology and Infectious Diseases. 2019; 25(11), 1347-1355. https://doi.org/10.1016/j.cmi.2019.04.025

8. Q Yi,D Cai,M Xiao, et al. Direct antimicrobial susceptibility testing of bloodstream infection on SlipChip. Biosensors and Bioelectronics. 2019;135:200-207. ://doi.org/10.1016/j.bios.2019.04.003

9. Morteza A, Benyamin D, Nguyen Ann V, et al. Biological small-molecule assays using gradient-based microfluidics. Biosensors \& bioelectronics. 2021; 178. ://doi.org/10.1016/j.bios.2021.1130381

10. Volbers D, Stierle VK., Ditzel KJ, et al. Interference Disturbance Analysis Enables Single-Cell Level Growth and Mobility Characterization for Rapid Antimicrobial Susceptibility Testing. Nano letters. 2019; 19(2): 643-651. ://doi.org/10.1021/acs.nanolett.8b02815

11. Huschek $\mathrm{D}$, Witzel $\mathrm{K}$. Rapid dereplication of microbial isolates using matrix-assisted laser desorption ionization time-of-flight mass spectrometry: A mini-review. Journal of Advanced Research. 2019; 19: 99-104. ://doi.org/10.1016/j.jare.2019.03.007 
12. Lau Anna F, Wang Honghui, Weingarten Rebecca A, et al. A rapid matrix-assisted laser desorption ionization-time of flight mass spectrometry-based method for single-plasmid tracking in an outbreak of carbapenem-resistant Enterobacteriaceae. Journal of clinical microbiology. 2014; 52(8):2804-12. ://doi.org/10.1128/JCM.00694-14

13. Youn Jung-Ho, Drake Steven K, Weingarten Rebecca A, et al. Clinical Performance of a MatrixAssisted Laser Desorption lonization-Time of Flight Mass Spectrometry Method for Detection of Certain blaKPC-Containing Plasmids. Journal of clinical microbiology. 2016; 54(1):35-42. ://doi.org/10.1128/JCM.01643-15

14. Miriam C,Markus k, Jörg G, et al. A Full MALDI-Based Approach to Detect Plasmid-Encoded KPCProducing Klebsiella pneumoniae. Frontiers in Microbiology. 2018; 9, 2854. ://doi.org/10.3389/fmicb.2018.02854

15. Hu Yan-Yan, Cai Jia-Chang, Zhou Hong-Wei et al. Rapid detection of porins by matrix-assisted laser desorption/ionization-time of flight mass spectrometry. Frontiers in Microbiology. 2015; 6, 784. ://doi.org/10.3389/fmicb.2015.00784

16. Jinfeng $B$,Yating $M, M e n g s h a n ~ D$, et al. Preliminary exploration on the serum biomarkers of bloodstream infection with carbapenem-resistant Klebsiella pneumoniae based on mass spectrometry. Journal of clinical laboratory analysis. 2021;://doi.org/10.1002/jcla.23915

17. Walter F, Lelio B, Cosmeri R, et al. Detection of Antibiotic-Resistance by MALDI-TOF Mass Spectrometry: An Expanding Area. Frontiers in Cellular and Infection Microbiology. 2020; 10, 572909. ://doi.org/10.3389/fcimb.2020.572909

18. C Sakarikou,M Ciotti,C Dolfa, et al. Rapid detection of carbapenemase-producing Klebsiella pneumoniae strains derived from blood cultures by Matrix-Assisted Laser Desorption Ionization-Time of Flight Mass Spectrometry (MALDI-TOF MS). BMC Microbiology. 2017; 17(1):54. ://doi.org/10.1186/s12866-017-0952-3

19. WC Mörschbächer,FGd Ross,CMd Santos, et al. Establishing a quantitative index of meropenem hydrolysis for the detection of KPC- and NDM-producing bacteria by MALDI-TOF MS. Journal of Microbiological Methods. 2021; 187, 106268. ://doi.org/10.1016/j.mimet.2021.106268

20. Lasserre C, De Saint Martin Luc, Cuzon G, et al. Efficient Detection of Carbapenemase Activity in Enterobacteriaceae by Matrix-Assisted Laser Desorption lonization-Time of Flight Mass Spectrometry in Less Than 30 Minutes. Journal of clinical microbiology. 2015; 53(7):2163-71. ://doi.org/10.1128/JCM.03467-14

21. Marina O, EvaandB Germán G. Rapid Detection of KPC-Producing Enterobacterales Susceptible to Imipenem/Relebactam by Using the MALDI-TOF MS MBT STAR-Carba IVD Assay. Frontiers in microbiology. 2020; 11: 328. ://doi.org/10.3389/fmicb.2020.00328

22. Christoph L, Sören S, Jette J, et al. Quantitative matrix-assisted laser desorption ionization-time of flight mass spectrometry for rapid resistance detection. Journal of clinical microbiology. 2014; 52(12):4155-62. ://doi.org/10.1128/JCM.01872-14 
23. Idelevich EA, Sparbier K, Kostrzewa M, et al. Rapid detection of antibiotic resistance by MALDI-TOF mass spectrometry using a novel direct-on-target microdroplet growth assay. Clinical microbiology and infection: the official publication of the European Society of Clinical Microbiology and Infectious Diseases. 2017; 24(7): 738-743. ://doi.org/10.1016/j.cmi.2017.10.016

24. Horseman TS, Lustik MB, Fong KSK. Rapid qualitative antibiotic resistance characterization using VITEK MS. Diagnostic Microbiology \& Infectious Disease. 2020; 97(4):115093.

://doi.org/10.1016/j.diagmicrobio.2020.115093

25. L Mengjiao,L Minxue,S Qifei, et al. Rapid antimicrobial susceptibility testing by matrix-assisted laser desorption ionization-time of flight mass spectrometry using a qualitative method in Acinetobacter baumannii complex. Journal of microbiological methods. 2018;153:60-65.

://doi.org/10.1016/j.mimet.2018.09.002

26. Maxson T,Taylor-Howell CL, Minogue TD. Semi-quantitative MALDI-TOF for antimicrobial susceptibility testing in Staphylococcus aureus. PLOS ONE. 2017; 12(8), e0183899. ://doi.org/10.1371/journal.pone.0183899

27. Abbey TC, Deak E. What's New from the CLSI Subcommittee on Antimicrobial Susceptibility Testing M100, 29th Edition. Clinical Microbiology Newsletter. 2019; 41(23).

://doi.org/10.1016/j.clinmicnews.2019.11.002<bi>.</bi>

28. Perez-Samper Gemma, Cerulus Bram, Jariani Abbas, et al. The Crabtree Effect Shapes the Saccharomyces cerevisiae Lag Phase during the Switch between Different Carbon Sources. mBio, 9(5). https://doi.org/10.1128/mBio.01331-18

29. Gang W, Guobin S, Yuanhong X. A Rapid Antimicrobial Susceptibility Test for Klebsiella pneumoniae Using a Broth Micro-Dilution Combined with MALDI TOF MS. Infection and drug resistance. 2021; 14 : 1823-1831. https://doi.org/10.2147/IDR.S305280

30. Caroline L, Guillaume G, Faiza M, et al. Comparison of Saramis 4.12 and IVD 3.0 Vitek MS MatrixAssisted Laser Desorption Ionization-Time of Flight Mass Spectrometry for Identification of Mycobacteria from Solid and Liquid Culture Media. Journal of clinical microbiology. 2017; 55(7): 2045-2054. https://doi.org/10.1128/JCM.00006-17

31. SK P, KJ E. The Inoculum Effect in the Era of Multidrug Resistance: Minor Differences in Inoculum Have Dramatic Effect on MIC Determination. Antimicrobial agents and chemotherapy. 2018; 62(8).https://doi.org/10.1128/AAC.00433-18

32. Tong Z, Zhou L, Li J, et al. In Vitro Evaluation of the Antibacterial Activities of MTAD in Combination with Nisin against Enterococcus faecalis. Journal of Endodontics. 2011; 7(8): 1116-20.

://doi.org/10.1016/j.joen.2011.03.020

33. Li C, Ding S, Huang Y, et al. Detection of AmpC $\beta$-lactamase-producing Gram-negative bacteria by matrix-assisted laser desorption/ionization time-of-flight mass spectrometry. Journal of Hospital Infection. 2018; 99(2): 200-207. ://doi.org/10.1016/j.jhin.2017.11.010

34. Monica $C$, Elena $M$, Federica $A$, et al. High-throughput genotyping of high-risk Human Papillomavirus by MALDI-TOF Mass Spectrometry-based method. The new microbiologica. 2015; 38(2): 211-23. 
35. Lieselotte V, Gemma PS, Bram C, et al. On the duration of the microbial lag phase. Current genetics. 2019; 65(3): 721-727. ://doi.org/10.1007/s00294-019-00938-2

Figures
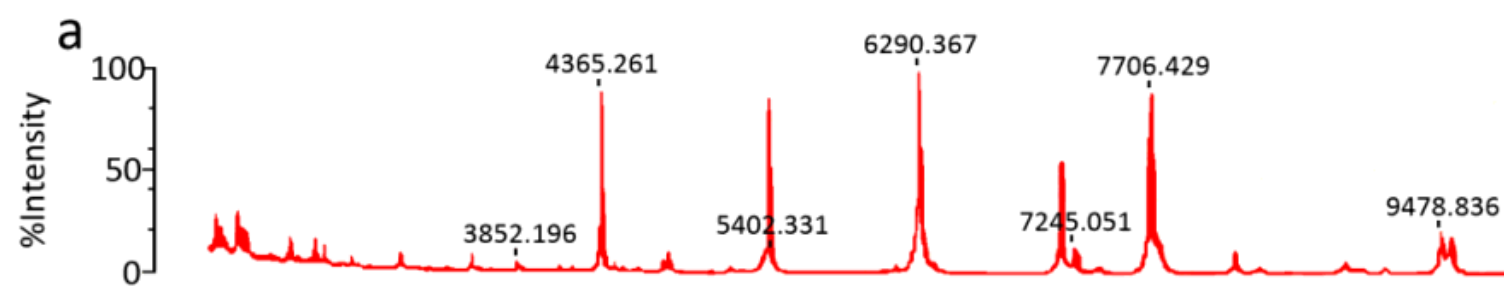

11877.539

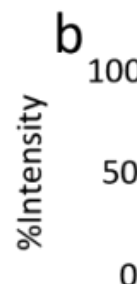

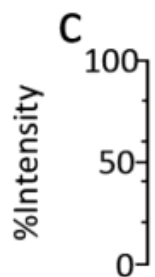
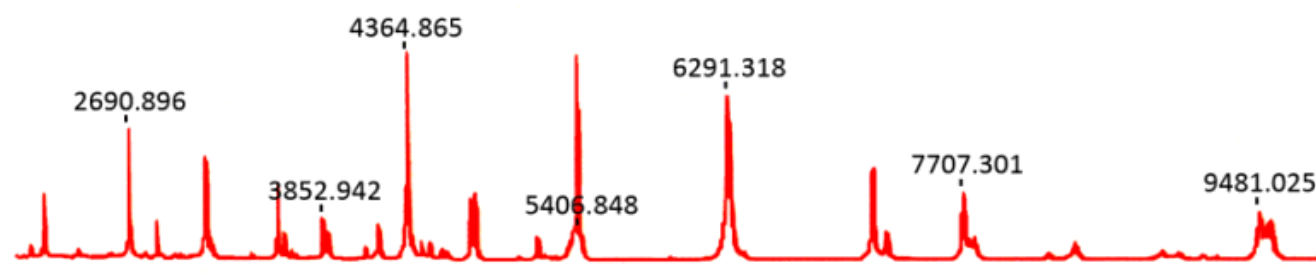

\section{Figure 1}

The differences in mass spectra of three sample preprocessing protocol. a Direct smear method. b Formic acid/ acetonitrile extraction method without washing: successfully identified as Klebsiella pneumoniae with the high background noise. c Formic acid/ acetonitrile extraction method with washing: successfully identified as Klebsiella pneumoniae with the mass spectra of higher quality. 

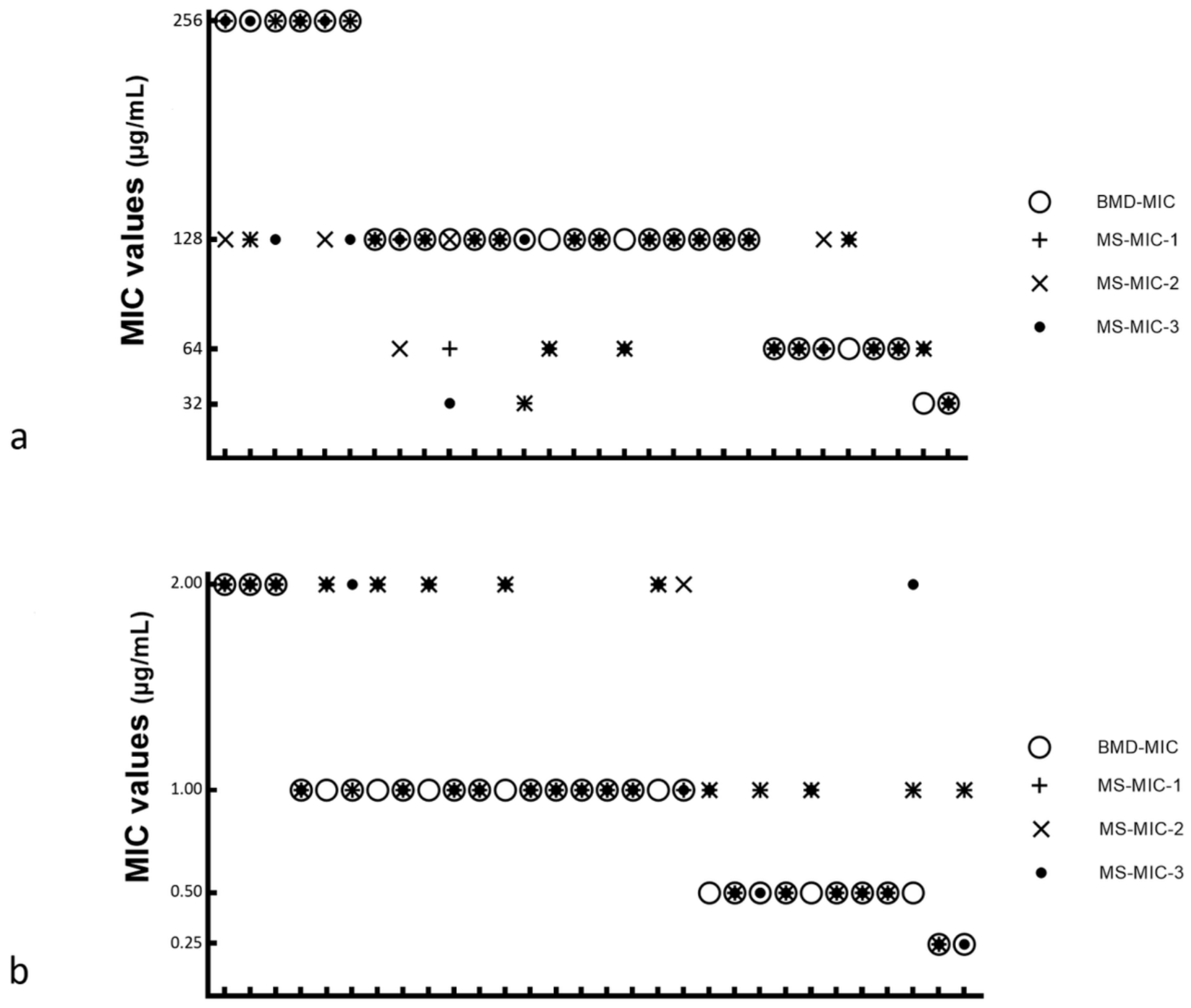

Figure 2

Reproducibility of MS-MIC values in CRKP (a) and CSKP (b) strains on different three days. 


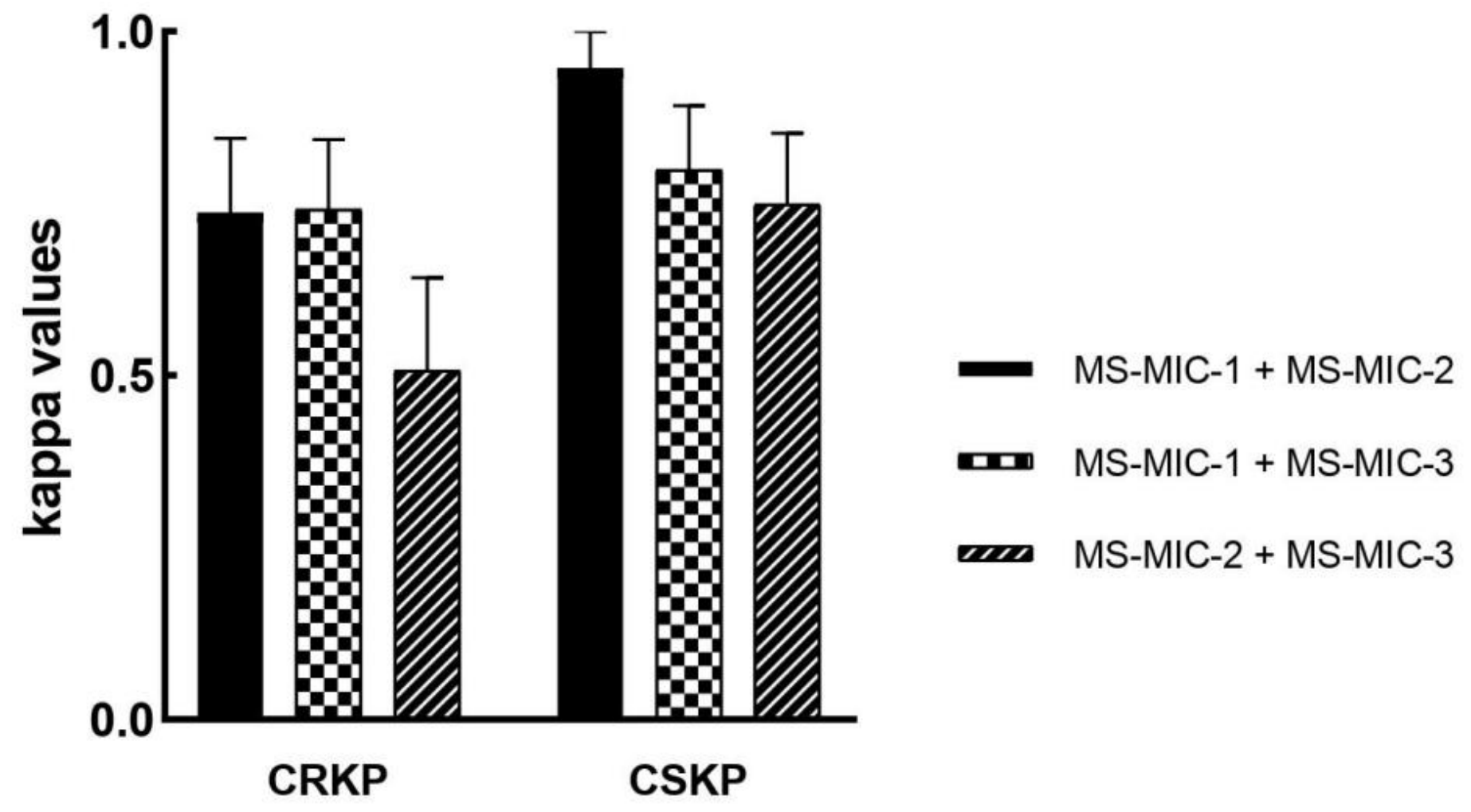

Figure 3

Consistency of three repeatable tests in CRKP and CSKP strains. 